\title{
Stochastic Diffusion Analysis for Sustainable Green Innovation

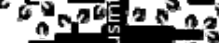

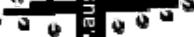 \\ auSMT
}

\section{Yenming J. Chen* and Chi-Ye Lan}

Department of Logistics Management, National Kaohsiung 1st University of Science \&Technology, Taiwan

(Received 24 June 2013; Accepted 15 August 2013; Published on line 1 December 2013)

*Corresponding author: yijchen@nkfust.edu.tw

DOI: $10.5875 / a u s m t . v 3 i 4.210$

Abstract: This study investigates the sustainability of green innovation under the influence of coopetitive diffusion. We focus on green innovations which repurpose discarded technology accessories for resale. Original manufacturers make every attempt to prevent the use of low-cost replacements. To encourage green innovation, incumbent market leaders need to be provided with business strategies which help them maximize profitability. Using a stochastic differential game from a Lotka-Volterra system, we predict a coopetitive diffusion path among participant stakeholders. We find that green innovation, combined with the positive effect of network externalities can indeed enhance total market growth. Factors such as product characteristics, product pricing, and compatibility protection strategies must interact properly for green innovation to be sustainable.

Keywords: Stochastic Lotka-Volterra system; stochastic Hamilton-Jacobi-Bellman equations; green innovation; coopetitive diffusion

\section{Introduction}

Some green innovations possess distinct advantages, whereas others are characterized by trade-offs. Governments often provide subsidies and legal protections to foster particularly risky green innovations. Despite these advantages, however, not all innovations survive over time. This study investigates the conditions for sustainability of such innovations and strategies for effective diffusion for remanufactured products in environments marked by both competition and cooperation.

Historically, innovations are more likely to fail than to succeed, e.g., cable modems, home circuit networks and personal digital assistants (PDAs). Fuel cell-powered vehicle technology has continuously promised various advantages, but public interest in this technology has been flagging. In 2003, the United States government proposed a Hydrogen Fuel Initiative, but reduced its scope by 2009 due to limited acceptance of the technology [1]. Superior technology does not ensure that an innovation will be successful and sustainable. Thomas Edison's success with the incandescent light bulb represents a classic example of survival amid alternative innovations: Contrary to common knowledge, the success of the light bulb owed as much to Edison's business strategy as to the technological invention [2]. At the time, Edison not only confronted strong collusion among gas utilities but, more importantly, a lack of electrical connections in households. Edison invested on an immature innovation among incumbent technologies, but managed to create sustainable growth through applying appropriate strategies [2].

Green innovation opportunities arise from creating alternatives to expensive, disposable accessories. Some companies sell their main products at a steep discount, only to recoup on subsequent sales of replacement or complementary parts, a model sometimes referred to as "razors and razorblades". Hewlett-Packard used to derive more than one-third of its profits from sales of ink and toner replacements for its printer products [3]. Given the extraordinarily high margins obtained from this lock-in effect, material and production efforts are usually 
disregarded. However, the environmental cost of disposal is transferred to the consumer. A green innovation is to refill disposed printer cartridges and sell the remanufactured products back to the consumer. The remanufacturing and the green value added fill the gap of environmental protection and provide the remanufacturer a surprisingly large profit. Although products with added green value enhance HP's public image and strengthen the company's position against competitors, HP is threatened by remanufactured cartridge providers, and litigates against them for patents infringement. This study investigates the sustainability of a green innovation practice under coopetitive product diffusion using cartridge remanufacturers as an example.

To effectively analyze strategic interactions, this study establishes a stochastic differential game based on a Lotka-Volterra system for predicting coopetitive diffusion patterns among green innovation stakeholders [6]. The model of market growth is based on a widely known diffusion formulation used in differential equations. A previous study [4] investigated the application of ecological models to business management and found that the ecological Lotka-Volterra model can be effectively used in predictions of business competition and management. Another study [5] analyzed market segmentation of two products and conducted experiments to verify the credibility of the Lotka-Volterra model. They showed that market evolutionary processes can be closely described and that business coopetition and Lotka-Volterra-modeled evolution are closely related. The same research [5] applied the Lotka-Volterra model in describing the relationship of market evolution between manufacturers and remanufacturers to master the changes of evolution between them under the interactions of different variables. In [6], sales volume diffusion dynamics was built based on the Lotka-Volterra coopetition model.

Discussions concerning remanufacturer intervention have focused on boycott tactics against remanufactured goods. A previous study [4] indicated that although firms cannot directly profit from reverse logistics, product-recycling systems need to intervene to prevent unnecessary competition from outrivaling. Given

YenmMing J. Chen is associate professor of Logistics Management, National Kaohsiung 1st University of Science and Technology, He received his PhD in Systems Science and Mathematics in 1998 at Washington University in St. Louis. His research interests include green supply chains, channel management, differential games, and cooperative games. He has published numerous articles on environmental management and strategic analysis.

Chi-Ye Lan was a graduate student in Logistics Management at the National Kaohsiung 1st University of Science \& Technology, He received his MBA in Logistics Management in 2009. the inevitable conflict from remanufacturers, many studies have begun to consider optimal business strategies from different aspects of competition, such as controlling remanufacturer intervention through price variation and product recycling [7-11].

However, recent studies have focused on the mutually beneficial relationship between the original and after-sales markets. Manufacturers could regard remanufactured products as a vehicle for green sales [12]. Following consumer preference in purchase intention, manufacturers could abandon first-season profits to increase production of remanufactured goods, as described in [1] where manufacturers face pricing issues of multi-seasonal products due to remanufacturer interference. Investigating the conflicts between both firms reveals the possibility of developing mutual sales aside from the preexisting competition. New trends in market development also stress conflict resolution through the application of product coopetition strategies.

Despite previous reports on the coopetitive characteristics of accessory markets, we still lack analytical models focused on the pattern of competitive diffusion. Given the sales mutualism of the two markets, simple competitive tactics are inappropriate in determining a manufacturer's best product strategy under real-life interactive sales conditions. This study demonstrates the mechanisms of strategic change in interactions between manufacturer and remanufacturer product sales. To elucidate the coopetitive strategic interactions among firms, this model not only considers the characteristics of a host product and its remanufactured market but consumer choice and preference of accessory products as well.

To understand how manufacturer product strategies affect volume changes in sales, we must first focus on two aspects: the characteristics of product demands and market diffusion systems. In terms of product properties, accessories are manufactured proprietarily because they require the joint usage of both the host and the accessory to function properly. Accessories only have value when used with their associated host products [13]. When an accessory is combined with its host product into a systemic product, the compatibility of an accessory with other brands influences consumer purchasing intention.

Systemic products have significant impacts on network externalities which, in turn, have direct or indirect impacts on consumers. With direct network externalities, consumer-perceived utility functions are directly influenced by other consumers of the same network [14]. With indirect network externalities, the utility of a consumer's choice is evaluated in terms of the 
investigated how variety of accessories affects consumer preferences, which is the central idea of indirect network externalities. Consumers benefit from network externalities through the combination of intrinsic product benefits and network external benefits [17]. intrinsic product benefits arise mainly from the consumer's own judgments, whereas external benefits are influenced by the number of users and accessories. The perceived value of consumer products rise with increased variety of complementary options, resulting in a positive feedback loop unique to network effects [14]. For example, in a host product's market, demand for accessories increases with the number of host product users, thus increased accessories further attract consumers through providing a variety of remanufacturing options.

As the installed base grows, increased popularity in the market provides consumers with better network externalities. For indirect network externalities, the growth of the installed base of systemic products is partly promoted by the accessory market. For example, during the lifespan of video games, product demands and market predominance can be controlled by price adjustments and supply diversification [18]. Market sales of CD players and CDs are mutually auxiliary [19]; increased variety in complementary software encourages purchases of hand-held electronic devices and increases product sales [20]. Therefore, firms that sell systemic products with such properties should adopt sales strategies that not only aim to boost basic consumer installation but consider complementary market support as well. Such strategies can induce positive feedback through network externality effects and reach product diffusion goals.

Through analysis of different market conditions, the predicted coopetitive diffusion pattern verified that the manufacturer could gain markedly higher profits by strategically allowing remanufacturer market intervention. We postulate that diffusion cannot be sustained if necessary factors such as product characteristics, product fragmentation and segmentation strategy do not properly interact in a competitive environment. Furthermore, we develop conditions for product sustainability in alternative markets.

The remainder of this paper is organized as follows. Section 2 reviews the spatial competition literature and demonstrates the importance of the current research. Section 3 discusses the modeling process. Section 4 presents behavior analysis conditions and shows our results on regional competition and diffusion. Section 5 concludes this paper by emphasizing the importance of cooperative competitors in the introduction of new products.

\section{Protocol Problem Description in Stochastic Differential Games}

Differential games, combining game theory with control theory, have been widely used in economics and management sciences [21] and are used in particular to investigate long-term dynamic negotiations among participants. A previous study [22] discussed the strategic interactions of pricing and advertising between suppliers and retailers using the Stackelberg model of a stochastic differential game. Considering uncertainty factors of consumer demand, further insights can be gained from analytical results that better conform to real-life situations.

This study considers a coopetitive game between a manufacturer and a remanufacturer that reproduces an knock-off accessory. The manufacturer first determines the prices of new items $p_{2}$ and compatibility or protection level $\theta$.The remanufacturer then determines the remanufactured accessory price. This study models the coopetition in a Stackelberg game with a symbiotic Lotka-Volterra stochastic system and derives the solution in terms of stochastic Hamilton-Jacobi-Bellman (HJB) equations.

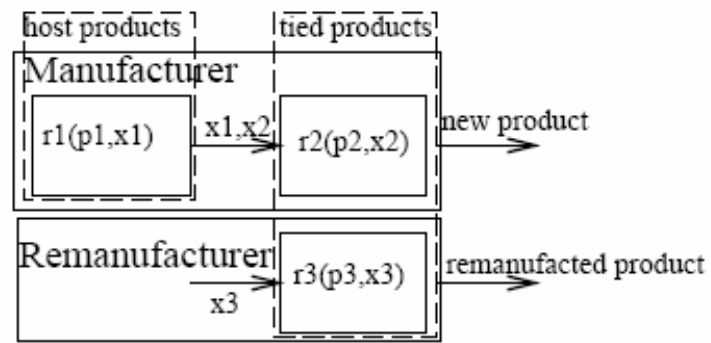

Figure 1.Conceptual model.

We denote $x_{1}$ and $x_{2}$ respectively as the sales quantities of the host products and the new accessory products, while $x_{3}$ represents the sales quantity for the reproduced accessories - a significant green innovation that reuses disposable materials from the original accessory. The host product is priced at $p_{1}$ and the two accessory products are respectively priced at $p_{2}$ and $p_{3}$. The model is shown in Figure 1. The manufacturer mainly earns profits from host device and accessory sales, which are respectively represented by $r_{1}\left(p_{1}, x_{1}\right)$ and $r_{2}\left(p_{2}, x_{2}\right)$. The manufacturer's prime costs are production cost $v_{1}\left(x_{1}\right)$ of the host product, production cost $v_{2}\left(x_{2}\right)$ of the accessory and compatibility control expenses $h_{i}(\theta)$ of the two accessories. Expenses for implementing control of host and accessory compatibility include costs for alternations to production procedures and product protection devices under the assumption that cost is an increasing convex function with respect to compatibility. 
increasing convex function with respect to compatibility.

In the competitive aspect of the game, both parties aim for maximal profits. Manufacturer profit is represented as follows:

$$
\begin{aligned}
\pi_{M}= & \int_{0}^{\infty}\left[r_{1}\left(p_{1}, x_{1}\right)-v_{1}\left(x_{1}\right)-h_{1}(\theta)+r_{2}\left(p_{2}, x_{2}\right) .\right. \\
& \left.-v_{2}\left(x_{2}\right)-h_{2}(\theta)\right] e^{\rho} d t
\end{aligned}
$$

The parameter $\rho$ represents the discount rate of the net present values of company profits. Remanufacturer profits arise from sales of remanufactured accessories, represented as $r_{3}\left(p_{3}, x_{3}\right)$. Remanufacturer prime costs consist of the product cost of the remanufactured accessory $v_{3}\left(p_{3}, x_{3}\right)$ and product compatibility cost $h_{3}(\theta)$, which includes expenses for product development to ensure compatibility. Remanufacturer gains result from the difference between remanufactured accessory sales and production and compatibility expenses, the function of which is assumed to be a decreasing convex function to compatibility. Remanufacturer profit is presented as follows:

$$
\pi_{R}=\int_{0}^{\infty} e^{-\rho t}\left[r_{3}\left(p_{3}, x_{3}\right)-v_{3}\left(x_{3}\right)-h_{3}(\theta)\right] d t
$$

In establishing an equilibrium coopetitive diffusion model that conforms to strategic interactions between both parties in the aforementioned product sales dynamic, the two most frequently used diffusion analysis models are the Bass and Lotka-Volterra models. Product coopetition relationships between firms are difficult to describe using the Bass model and many researchers have applied ecological models to compensate for its deficiencies.

The dynamics of competitive diffusion in this paper are based on a mutualism model. The equations incorporate additional stochastic terms to describe the uncertainty from the influence of other firms. Let $Q_{i}$, for $i=1,2,3$ represent the maximal market sales volume or the market saturation volume. The dynamics of three firms in terms of sales volumes are described as follows:

$$
\begin{gathered}
d x_{1}=\alpha_{1} x_{1}\left[\left(Q_{1}-x_{1}\right)+\delta\left(p_{2}, p_{3}, \theta\right)\left(x_{3}\right)\right] d t \\
+\sigma_{1}\left(x_{1}\right) d z(t) \\
d x_{2}=\alpha_{2} x_{2}\left[\begin{array}{l}
\left(Q_{2}-x_{2}\right)+e_{2} x_{1}- \\
f_{2}\left(p_{2}, p_{3}\right)\left(x_{3}-g_{2}(\theta)\right)
\end{array}\right] d t, \\
+\sigma_{2}\left(x_{2}\right) d z(t)
\end{gathered}
$$

$$
\begin{aligned}
d x_{3}= & \alpha_{3} x_{3}\left[\begin{array}{l}
\left(Q_{3}-x_{3}\right)+e_{3} x_{1}- \\
f_{3}\left(p_{2}, p_{3}\right)\left(x_{2}-g_{3}(\theta)\right)
\end{array}\right] d t \\
& +\sigma_{3}\left(x_{3}\right) d z(t)
\end{aligned}
$$

Where $\alpha_{i}$, for $i=1,2,3$ denotes the intrinsic growth rate in a market and is assumed to be a constant and uninfluenced by controlled variables; $\sigma\left(x_{i}\right)$ for $i=1,2,3$ is the variance term; the random variable $z(t)$ represents a standard Wiener process in a probability space $(\Omega, \mathrm{I}, P)$.

In the coopetitive process, two opposite forces from the rivalry partners influence the diffusion pattern of the products. Let $f_{i}$ be the degree to which new and remanufactured accessories cannibalize each other, and let $\delta\left(p_{2}, p_{3}, \theta\right)$ be the contribution of the positive network effect, which is attributed to the sales of green innovative accessories produced by a third party. The cannibalization effect is assumed to be a function of accessory prices $p_{2}$ and $p_{3}$ and is related to the extent of price difference. Variations to the price differences, $\left(k_{4}\left(p_{2}-p_{3}\right)\right)$ and $\left(k_{7}\left(p_{3}-p_{2}\right)\right)$, between the two products, may impact consumer purchases of remanufactured accessories, where $k_{4}$ and $k_{7}$ respectively represent the influence of price difference on market demands for new and remanufactured accessories.

The network effect $\delta\left(p_{2}, p_{3}, \theta\right)$ is influenced by the price difference $\left(k_{1}\left(p_{2}-p_{3}\right)\right)$ and the compatibility factor $\left(k_{2}(1-\theta)\right)$, where $k_{1}$ is similar to the meaning of $k_{4}$ and $k_{7}$, and $k_{2}$ represents the influence of compatibility on host product sales. $k_{3}$ is the degree of change in the accessory product market caused by that of the host product. Host product sales intuitively have a positive impact on accessory sales. The parameters $e_{2}$ and $e_{3}$ respectively represent sales benefits to new and remanufactured accessories.

The protection effect $g_{2}(\theta)$ represents the competition of the remanufactured accessory on the new accessory; conversely, $g_{3}(\theta)$ represents the opposite competition effect from the other direction. As protection slackens, compatibilities $k_{5}(1-\theta)$ and $k_{8}(1-\theta)$ increase, thereby boosting demand and supply of remanufactured accessories as well as demand for host products and new accessories, where $k_{5}$ denotes the effects of compatibility on new accessory product needs, and $k_{8}$ represents the effect of the host product market on demand for remanufactured accessory products.

\section{System Strategic Analysis}

In investigating the equilibrium of interactive strategies in the coopetition relationship, cannibalization 
increases in severity when substitutes can be found for original manufacturer products. Producers must secure market dominance to prevent remanufactured accessories from cannibalizing the profit of the original producer, especially if consumers are less subject to brand loyalty.

Firms often exhibit distinct competitive advantages in a market. Some firms enter the market early, quickly achieving dominance and leading the market by setting standards for all related products. Late entrants respond strategically according to the tactics of the market leaders firms and their own advantages. In this study, the original manufacturer leads the accessory market and the accessory remanufacturer must react optimally to the manufacturer's decisions. More importantly, the manufacturer has to maximize profits by determining business strategies appropriate to the expected remanufacturer response.

\section{Equilibrium pricing strategies for remanufacturer (follower)}

As the leader in the sequence of decisions, the manufacturer determines the price $p_{2}(\cdot)$ of the accessory product and the degree of compatibility $\theta(\cdot)$. The remanufacturer makes a corresponding decision $p_{3}(\cdot)$ according to the given tactics of the leading manufacturer. Hence, remanufacturer strategic control can be obtained as follows:

$$
V^{F}\left(x_{3}\right)=\max _{p_{3}}\left\{\begin{array}{c}
E \int_{0}^{\infty}\left[r_{3}\left(p_{3}^{F}, x_{3}\right)-\right. \\
\left.v_{3}\left(x_{3}\right)-h_{3}(\theta)\right] d t
\end{array}\right\},
$$

such that

$$
\begin{aligned}
x_{3}^{F}(t)= & \alpha_{3} x_{3}\left(Q_{3}-x_{3}\right)+e_{3} x_{3} \\
& -f_{3}\left(p_{2}, p_{3}\right)\left(x_{2}-g_{3}(\theta)\right)+\sigma_{3}\left(x_{3}\right) d z(t)
\end{aligned}
$$

According to the stochastic HJB equation, defining remanufacturer costates (including remanufactured accessory sales variation, $\alpha_{3} x_{3}\left(Q_{3}-x_{3}\right)+e_{3} x_{3}-f_{3}\left(p_{2}, p_{3}\right)$ $\left(x_{2}-g_{3}(\theta)\right)$, costate $V_{x 3}$ and costate $V_{x_{3} x_{3}}$ of its stochastic term $\sigma\left(x_{3}\right)$ and combining remanufacturer objective (6) and state variable (7) lead to the following HJB equation:

$$
r V^{F}=\max \left\{\begin{array}{l}
{\left[r_{3}-v_{3}-h_{3}\right]+V_{x_{3}}^{F} x_{3}^{F}} \\
+V_{x_{3} x_{3}}^{F} \frac{1}{2} \sigma^{2}\left(x_{3}\right) V_{x_{3} x_{3}}
\end{array}\right\} .
$$

Under the specific strategy imposed by the manufacturer, the remanufacturer response strategies must fulfill the first-order condition for the right-hand side (RHS) of (8):

$$
\frac{\partial R H S}{\partial p_{3}}=0
$$

Remanufacturer strategic response $p_{3}^{F}$ for the accessory price $p_{2}^{L}(\cdot)$ and compatibility $\theta(\cdot)$ given by the manufacturer may be obtained using the first-order condition. Let $p_{3}^{F}$ be replaced with $\mathrm{R}$ and substituted back into (8) to yield an $\mathrm{HJ}$ equation (10) with only state and co-state variables.

$$
r V^{F}=\left[r_{3}-v_{3}-h_{3}\right]+V_{x_{3}}^{F} x_{3}^{F}+V_{x_{3} x_{3}}^{F} \frac{1}{2} \sigma_{3}^{2} V_{x_{3} x_{3}}^{F} .
$$

Equilibrium price and compatibility strategies for manufacturer (leader)

The leading manufacturer adjusts its equilibrium controls $p_{2}^{F}$ and $\theta^{F}$ according to market changes when it perceives the remanufacturer response $p_{3}^{F}$ under the conditions of the preexisting accessory price $p_{2}^{L}(\cdot)$ and compatibility $\theta(\cdot)$. In such an interaction, the remanufacturer counter-price strategy $R$ must replace $p_{3}^{F}$. The problem for the equilibrium control of the manufacturer can be expressed as follows:

$$
V^{L}\left(x_{1}, x_{2}\right)=\max _{p_{2}, \theta} E\left\{\int_{0}^{\infty}\left[\begin{array}{l}
r_{1}\left(p_{1}, x_{1}\right)-v_{1}\left(x_{1}\right) \\
-h_{1}(\theta)+r_{2}\left(p_{2}, x_{2}\right) \\
-v_{2}\left(x_{2}\right)-h_{2}(\theta)
\end{array}\right] d t\right\}
$$

Substitute $P_{3}^{F}$ with $R$ in sales dynamics (12), (13), and (14) as follows:

$$
x_{1}^{L}=\alpha_{1} x_{1}\left[\left(Q_{1}-x_{1}\right)+\delta\left(p_{2}, R, \theta\right) x_{3}\right]+\sigma\left(x_{1}\right) d z(t),
$$

$$
\begin{aligned}
& x_{2}^{L}=\alpha_{2} x_{2}\left[\begin{array}{l}
\left(Q_{2}-x_{2}\right)+e_{2} x_{1} \\
-f_{2}\left(p_{2}, R\right)\left(x_{3}-g_{2}(\theta)\right)
\end{array}\right]+\sigma\left(x_{2}\right) d z(t), \\
& x_{3}^{L}=\alpha_{3} x_{3}\left[\begin{array}{l}
\left(Q_{3}-x_{3}\right)+e_{3} x_{1} \\
-f_{3}\left(p_{2}, R\right)\left(x_{2}-g_{3}(\theta)\right)
\end{array}\right]+\sigma\left(x_{3}\right) d z(t),
\end{aligned}
$$

From the objective (11) and sales dynamics (12), (13), and (14), the following HJB equation is generated:

$$
r V^{L}\left(x_{1}, x_{2}\right)=\max \left\{\begin{array}{l}
{\left[r_{1}-v_{1}-h_{1}+r_{2}-v_{2}-h_{2}\right]+V_{x_{1}} x_{1}^{L}} \\
+V_{x_{2}} x_{2}^{L}+V_{x_{3}} x_{3}^{L}+V_{x_{1} x_{1}} \frac{\sigma_{1}^{2} V_{x_{1} x_{1}}}{2} \\
+V_{x_{2} x_{2}} \frac{\sigma_{2}^{2} V_{x_{2} x_{2}}}{2}+V_{x_{3} x_{3}} \frac{\sigma_{3}^{2} V_{x_{3} x_{3}}}{2}
\end{array}\right\}
$$

Remanufacturer responsive strategies must fulfill the first-order condition of the RHS of (15) for the equilibrium control in HJB equations under the specific strategy imposed by the manufacturer: 


$$
\begin{aligned}
& \frac{\partial R H S}{\partial p_{2}^{L}}=0, \\
& \frac{\partial R H S}{\partial \theta^{L}}=0,
\end{aligned}
$$

We can solve for the manufacturer equilibriums $p_{2}^{L}(\cdot)$ and $\theta^{L}(\cdot)$ by using the first-order conditions in (16) and (17). Let $L 1$ and $L 2$ respectively replace $p_{2}^{L}(\cdot)$ and $\theta^{L}(\cdot)$, and substitute the results into (15) to obtain an $\mathrm{HJB}$ equation (18) that involves only state and costate variables as follows:

$$
\begin{aligned}
r V^{L}\left(x_{1}, x_{2}\right)= & {\left[r_{1}-v_{1}-h_{1}+r_{2}-v_{2}-h_{2}\right] } \\
& +V_{x_{1}} x_{1}^{L}+V_{x_{2}} x_{2}^{L}+V_{x_{3}} x_{3}^{L}+V_{x_{1} x_{1}} \frac{\sigma_{1}^{2} V_{x_{1} x_{1}}}{2} . \\
& +V_{x_{2} x_{2}} \frac{\sigma_{2}^{2} V_{x_{2} x_{2}}}{2}+V_{x_{3} x_{3}} \frac{\sigma_{3}^{2} V_{x_{3} x_{3}}}{2}
\end{aligned}
$$

Systems of equations are established from (10) and (18) to solve for the costate variables of both firms and further evaluate the manufacturer equilibriums $p_{2}^{*}$ and $\theta^{*}$ as well as the remanufacturer equilibrium $p_{3}^{*}$. However, the costate variables of the model in this study are complex and difficult to solve explicitly. Hence, sales dynamics are divided into deterministic and stochastic parts. The Pontryagin maximum principle can be adopted to solve the deterministic part, and costate variables are entered into the stochastic differential equation in Matlab for equilibrium solutions in a deterministic differential game. The first-order conditions derived from HJB equations are then substituted into a stochastic differential game to obtain all related solutions.

\section{Single-manufacturer market analysis}

For comparison, we started the analysis by modeling a market without remanufacturer intervention. In the case of a monopolist market, an optimal solution was obtained. Remanufacturer-related coefficients were set to zero to remove the influence of remanufacturer. The sales volumes of the host product and the new accessory were initially assumed to be equal $\left(x_{1}^{0}=x_{2}^{0}\right)$.

We calculated the expected manufacturer profit by conducting 30 simulation runs under varying market conditions. Related parameter settings are shown in Table 1. The level of the beneficial effects of host product sales on accessory sales $\left(k_{3}\right)$ and the host product intrinsic market growth rate $\left(\alpha_{1}\right)$ were respectively set to 0.5 and 0.25 . The accessory intrinsic growth rate $\left(\alpha_{2}\right)$ was determined by simulation because we let the rate be randomly chosen within the range $[0.25,0.4]$.

Selected results from the 30 Monte Carlo simulations are shown in Figure 2. The optimal pricing strategy to attract customers is to adopt a lower accessory price $\left(p_{2}\right)$ to penetrate the market and then gradually return to the market price. The compatibility $\theta$ is unnecessarily without the intervention of the remanufacturer. We demonstrate that a proper strategy can always increase profit in a monopolist market.

Table 1. Parameters for the case of a single manufacturer.

\begin{tabular}{ccc}
\hline Parameters & The manufacturer & The remanufacturer \\
\hline$\alpha$ & $\alpha_{1}=0.25$, & $\alpha_{3}=0$ \\
$x^{0}$ & $\alpha_{2}=[0.25,0.4]$ & $x_{3}^{0}=0$ \\
$\mathrm{Q}$ & $x_{1}^{0}=2, x_{2}^{0}=2$ & $Q_{3}=0$ \\
$\mathrm{k}$ & $Q_{1}=6, Q_{2}=6$ & $k_{5}=k_{6}=k_{7}=k_{8}=0$ \\
& $k_{1}=k_{2}=k_{4}=0$, & \\
$\sigma$ & $k_{3}=0.5$ & $\sigma\left(x_{3}\right)=0$ \\
\hline
\end{tabular}
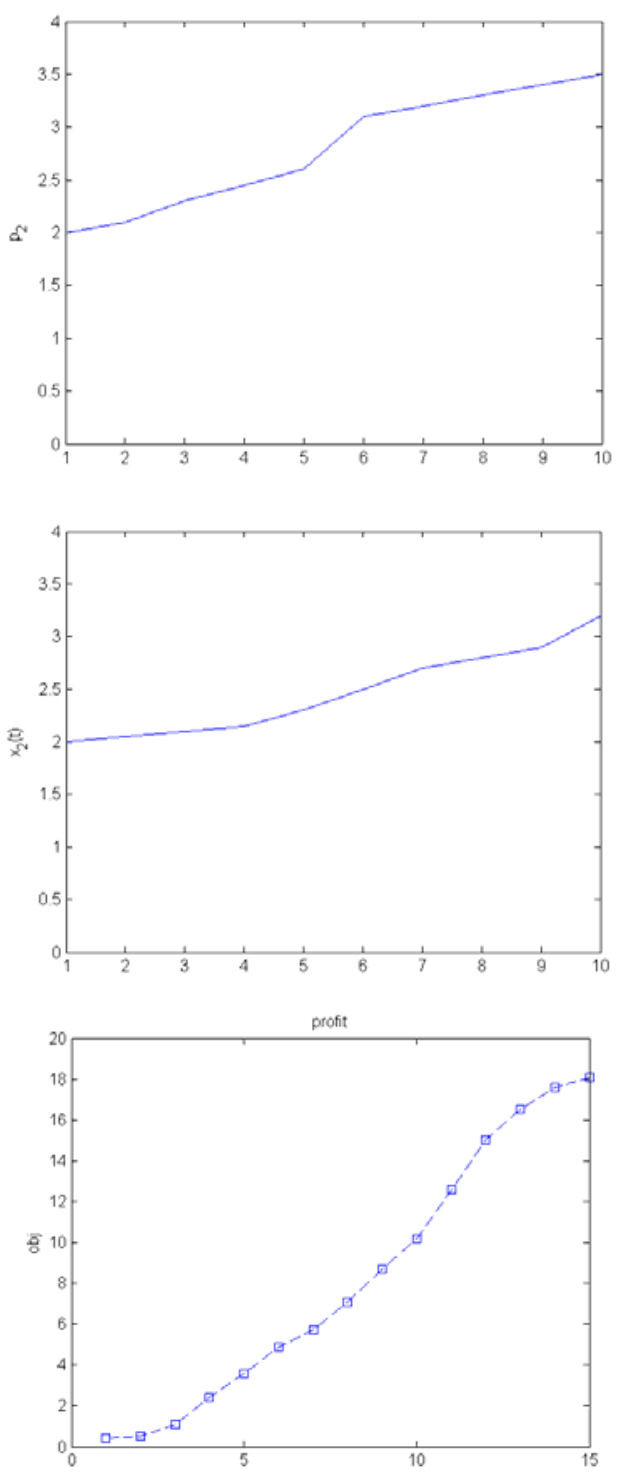

Figure 2. Optimal pricing strategies, sales dynamics and profits for a single-manufacturer market. 


\section{Manufacturer and remanufacturer mutualism}

For coexisting markets, the manufacturer produces both the host and the accessory products, whereas the remanufacturer competes by selling a refilled accessory product. In this situation, we are interested in the equilibrium strategies of each player.

The initial sales volume of the new accessories was assumed to be higher than that of the remanufactured accessories $\left(x_{2}^{0}>x_{3}^{0}\right)$. The intrinsic growth rate of the former was assumed to exceed that of the latter $\left(\alpha_{2}>\alpha_{3}\right)$. In previous single-manufacturer scenarios, $\alpha_{1}$ was set to 0.25 for benchmarking, and in this simulation scenario $\alpha_{2}$ and $\alpha_{3}$ were assumed to be random numbers within $[0.25,0.4]$ and $[0.1,0.25]$, respectively. Given that the remanufactured accessory is limited by the supply of the new accessory, $\alpha_{3}$ is always smaller than $\alpha_{2}$. A series of 30 simulations was conducted to obtain the expected profit of the manufacturer. Under the premise of maximal rationality, when the two producers secure approximately the same share, consumers become more sensitive to differences in accessory prices but less sensitive to accessory compatibility. When the two markets of accessories are imbalanced, the manufacturer can improve its profits if moderate intervention by the remanufacturer is allowed by introducing proper pricing and compatibility strategies.

To increase profits, the manufacturer can allow the remanufacturer to become moderately involved in the accessory market. Moderate involvement of the remanufacturer can widen accessory variety, thereby promoting host product usage and simultaneously increasing new demand for remanufactured accessories. Simulation results show that, given significant superiority to other suppliers, the original producers can attain higher profits compared than that of individual sales by the allowance strategy. This result is consistent with previous findings in [23].

A significant difference was assumed between the beneficial effects of the host products and remanufactured accessories. The former was assumed to exceed the latter $\left(k_{3}=0.5>k_{6}=0.25\right)$. Moreover, the effects of price differences on new accessory sales were assumed to be greater than for remanufactured accessory sales $\left(k_{4}=0.6>k_{7}=0.4\right)$. In this simulation scenario, we examined the effect of the remanufacturer on the sales of the original manufacturer with competitive diffusion in the market considered. The related parameters are shown in Table 2 .

In this simulation scenario, the intrinsic growth rate of the new accessory $\left(\alpha_{2}\right)$ was set to 0.3506 (as in the new product manufacturer simulation). Random settings ( $\alpha_{2}=0.2231$ ) were selected by a Monte Carlo
Table2. Co-existence of manufacturer and remanufacturer.

\begin{tabular}{ccc}
\hline Parameter & Manufacturer & Remanufacturer \\
\hline$\alpha$ & $\alpha_{1}=0.25$, & $\alpha_{3}=[0.1,0.25]$ \\
$x^{0}$ & $\alpha_{2}=[0.25,0.4]$ & $x_{3}^{0}=1.2$ \\
\hline
\end{tabular}

test, and the effects of the remanufacturer market entrance on market sales dynamics were examined. Observation of equilibrium strategies for both firms demonstrates that manufacturers with greater market advantage can initially set higher accessory prices $\left(p_{1}\right)$. Given remanufacturers' relative market weakness, they usually set low prices $\left(p_{2}\right)$ to extend market reach. However, the equilibrium strategies eventually suggest symbiotic growth in both sales thus, rather than completely barring remanufacturers, firms can instead create a win-win situation through properly adjusting business strategies.

The equilibrium manufacturer strategies and adjustments suggest that new accessory prices should follow low-cost penetration strategies to attract consumers and that compatibility protection should be lowered gradually. The implied purpose is to allow more remanufactured accessories to enter the market by clearing the barrier of hardware protection, thus boosting customer usage by the spillover effects of all accessory products. These strategies suggest that new accessory prices can gradually increase as market demand grows. The remanufacturer should closely watch the strategies of the original manufacturer. The best responsive strategy for a remanufacturer is to maintain a constant difference in price to avoid retaliation on the part of the manufacturer by tightening the protection level $\theta(\cdot)$ for new accessory products. That is, the remanufactured green innovator should follow the pricing trend of the original producer and gradually raise the sales price of remanufactured accessories (as shown in Figure 3).

As the prices of remanufactured accessories gradually increase, the cannibalization effect decreases. As shown in the subplots of Figure 3, the host product sales rose from an initial volume of 2 to a final volume of 3 and the total sales volume increased from 3.4 to 3.8 , respectively, when the remanufactured accessories were allowed to co-exist with the original accessories. New accessory sales grew from 2 to 2.8 in subsequent stages. Remanufactured accessory sales increased from 1.2 to 1.4 because of the network effect of the growth of the accessory market. Compared with the previous single-manufacturer scenario, the introduction of the remanufactured accessories increased manufacturer profits, as shown in Figure 4. 

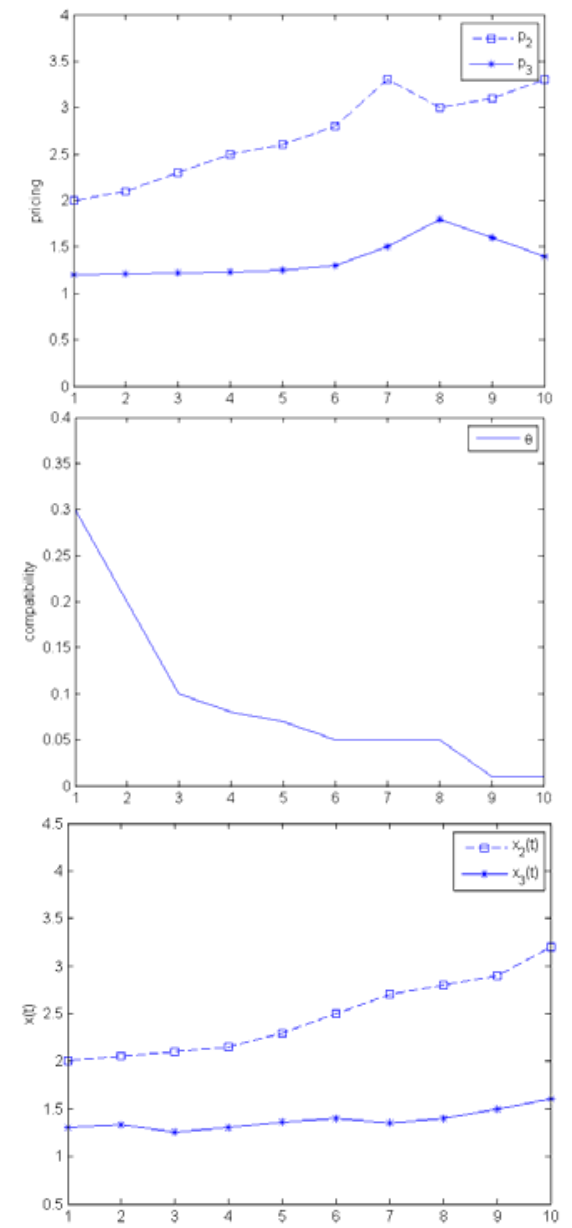

Figure 3.Equilibrium pricing, compatibility strategies and sales dynamics in market mutualism.

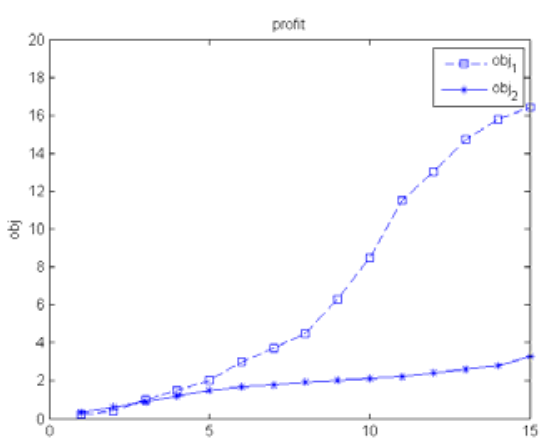

Figure 4.Profit increments in market mutualism.

To determine whether the remanufacturer assists in the expansion and development of the total market, 30 Monte Carlo simulation results were collected for a statistical test to determine whether a significant difference in manufacturer expected returns is indicated by the presence or absence of cooperative firms. The hypothesis tests whether the profit of the monopolist manufacturer is higher than that of the co-existing one. The results of the t-test in manufacturer profit are presented in Table 3.

Table 3. t-test of manufacturer profit.

\begin{tabular}{cccc}
\hline $\begin{array}{c}\text { Single } \\
\text { manufacturer }\end{array}$ & Coexistence & $\begin{array}{c}\mathrm{t} \\
\text { Value }\end{array}$ & $\begin{array}{c}\mathrm{P}(\mathrm{T} \leq \mathrm{t}) \\
\text { single tail }\end{array}$ \\
\hline 19.46 & 31.05 & -15.61 & $5.99 \mathrm{E}-16$ \\
\hline
\end{tabular}

Results indicate that the null hypothesis should be rejected at a significance level of $95 \%$, and a $p$-value smaller than 0.05; that is, the remanufacturer involvement significantly benefits the manufacturer profits. Test results verify that, in the presence of asymmetric market competence and strategy-adjusting abilities, the moderate allowance of remanufacturer intervention can increase demand for the original products.

\section{Sensitivity analysis in simulation scenarios}

This subsection provides further analysis to establish relationships among various market factors, such as market asymmetry and customer sensitivity to compatibility. We designed a nine-scenario simulation experiment similar to the design of settings in Table 2. The parameter settings for this subsection are listed in Tables 4 and 5:

Table 4. Basic parameter settings for the asymmetrical market.

\begin{tabular}{|c|c|c|c|c|c|c|c|}
\hline & $x_{1}^{0}$ & $x_{1}^{0}$ & $\alpha_{1}$ & $\alpha_{2}$ & $k_{4}$ & $k_{6}$ & $k_{2}$ \\
\hline $\begin{array}{l}x_{2}^{0}>x_{3}^{0}, \\
\alpha_{1}>\alpha_{2} \\
k_{6}<k_{4}\end{array}$ & 2 & 1.2 & 0.3506 & 0.2231 & 0.25 & 0.5 & 0.3 \\
\hline $\begin{array}{l}x_{2}^{0}>x_{3}^{0}, \\
\alpha_{1}<\alpha_{2}, \\
k_{6}>k_{4}\end{array}$ & 2 & 1.2 & 0.2231 & 0.3506 & 0.5 & 0.25 & 0.3 \\
\hline $\begin{array}{l}x_{2}^{0}>x_{3}^{0}, \\
\alpha_{1}<\alpha_{2}, \\
k_{6}<k_{4}\end{array}$ & 2 & 1.2 & 0.2231 & 0.3506 & 0.25 & 0.5 & 0.3 \\
\hline
\end{tabular}

Table 5. Simulations for the nine scenarios in market conditions.

\begin{tabular}{cccc}
\hline Degree of asymmetry & Low sensitivity & Medium sensitivity & High sensitivity \\
\hline $\operatorname{Low}\left(x_{1}^{0}=1.6, x_{2}^{0}=1.6, x_{3}^{0}=1.2\right)$ & $k_{2}^{0}=0.1$ & $k_{2}^{0}=0.3$ & $k_{2}^{0}=0.5$ \\
$\operatorname{Med} x_{1}^{0}=2.0, x_{2}^{0}=2.0, x_{3}^{0}=1.2$ & $k_{2}^{0}=0.1$ & $k_{2}^{0}=0.3$ & $k_{2}^{0}=0.5$ \\
$\operatorname{High}\left(x_{1}^{0}=2.4, x_{2}^{0}=2.4,, x_{3}^{0}=1.2\right)$ & $k_{2}^{0}=0.1$ & $k_{2}^{0}=0.3$ & $k_{2}^{0}=0.5$ \\
\hline
\end{tabular}


Scenarios for sensitivity analysis of market mutualism were extended, and the equilibrium strategies were investigated. We assumed that degrees of high, medium, and low market asymmetry exist in the initial market shares of the manufacturer and the remanufacturer. Sensitivity to compatibility was also considered at high, medium, and low levels. Given particular degrees of asymmetry and sensitivity, the manufacturer then determines equilibrium strategies under which the rival remanufacturer exerts its own equilibrium strategies. Nine scenarios were compared and analyzed in the experiment.

Manufacturer equilibrium pricing and compatibility strategies can be determined in each scenario with stochastic simulations. The manufacturer profits in different scenarios are shown in Table 6.

Table 6.Manufacturer profits for the nine market conditions.

\begin{tabular}{cccc}
\hline $\begin{array}{c}\text { Degree of } \\
\text { asymmetry }\end{array}$ & $\begin{array}{c}\text { Low } \\
\text { sensitivity }\end{array}$ & $\begin{array}{c}\text { Medium } \\
\text { sensitivity }\end{array}$ & $\begin{array}{c}\text { High } \\
\text { sensitivity }\end{array}$ \\
\hline LowAsymm & 5.11 & 4.42 & 41 \\
MedAsymm & 20.56 & 20.0 & 22.92 \\
HighAsymm & 52.47 & 53.26 & 55.48 \\
\hline
\end{tabular}

Given manufacturer and remanufacturer mutualism, high consumer sensitivity to compatibility decreases total sales when market asymmetry is low. This is because an increase in consumer sensitivity to compatibility represents a greater degree of cannibalism than of sales mutualism in the market. The reason is that the manufacturer does not completely predominate the market, causing a decline in total sales profit. In addition, sensitivity to compatibility significantly affects the manufacturer. When sensitivity is low, new product sales suffer less from the cannibalization of the remanufactured products. However, when sensitivity is moderate, cannibalization negatively affects the competitive advantages of the manufacturer, and the profit decreases slightly. When sensitivity increases, the dual effects of market dominance and sales mutualism enable the manufacturer to improve its profit.

Simulation analysis of different market conditions in Figure 5 shows that manufacturer equilibrium strategies are greatly influenced by the degree of market asymmetry and the sensitivity characteristics. The simulation result demonstrates that compatibility protection must be strengthened as consumer sensitivity increases. When such sensitivity is maintained at a moderate level, competition and the benefits of mutualism cancel each other out. When sensitivity is low, original manufacturers should tighten product protection during early sales stages to prevent the cannibalization of the remanufacturing market from overriding the benefits of sales mutualism. In addition, despite the occurrence of protectionism under conditions of high sensitivity of compatibility, manufacturers can gradually lower the level of protection during the product's lifespan and tactically allow the entry of remanufacturer to increase their own profits.
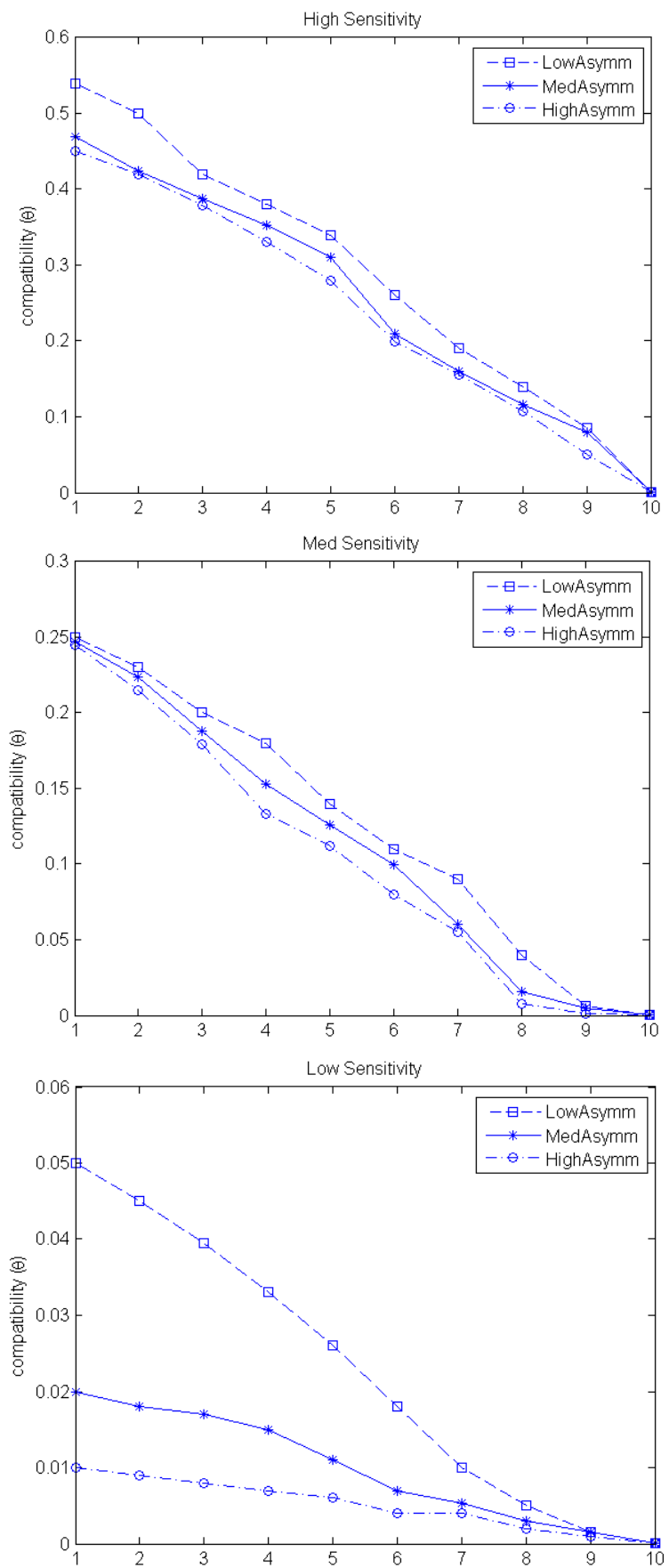

Figure 5. Manufacturer equilibrium compatibility control paths for the nine scenarios.

\section{Conclusion}

This study combines the evolutionary part of market growth and the equilibrium part of strategic manipulation in a coopetitive diffusion model. We conclude that a green innovation can be sustainable if all parties can react strategically according to the market 
dynamics resulting from the response of all parties. We successfully link the temporal diffusion to a green innovation in a cooperative analysis in a stochastic differential game, which has been overlooked in other studies.

Given sales conflict over time, this study proposes that manufacturers can manipulate their advantages and strategies to achieve total sales growth and enter a win-win market situation. In addition, market asymmetry and consumer sensitivity to compatibility are key strategic issues. Given equal market shares, original manufacturers suffer from severe cannibalization from remanufacturers. Thus, original manufacturers should adopt higher compatibility protection strategies to prevent loss of sales.

As the market changes in complex competitive interactions, enterprises must not be limited to conventional responses to competition but rather boost overall market demand by strategically cooperating with other firms. The influence of accessory remanufacturers should also be considered. Interactions among all accessory manufacturers often change, thereby influencing the best strategies developed by each company. The essence of competition has changed because the new green innovation changes the market structure in the original market. Strategies which resist remanufacturer involvement entail higher expenditures for product protection, and may limit opportunities for product sales growth due to the lack of diversity in the accessory market. This situation is undoubtedly adequate for manufacturers that strive for higher market share and maximal profits.

This paper provides a new approach to the dynamic coopetitive diffusion for green innovation. Although the cooperative diffusion model considers factors which influence product diffusion (e.g., consumer preferences, price differences and network externalities), the study is subject to certain limitations. Future studies may develop a three-firm model to make the two-party coopetitive diffusion model presented here better conform to real-world scenarios. Collection of market data and appropriate quantification of analytical verification may also widen the scope of this model.

\section{References}

[1] P. E. Meyer and J. J. Winebrake, "Modeling technology diffusion of complementary goods: The case of hydrogen vehicles and refueling infrastructure," Technovation, vol. 29, no. 2, pp. 77-91, 2009. doi: 10.1016/j.technovation.2008.05.004
[2] A. B. Hargadon and Y. Douglas, "When innovations meet institutions: Edison and the design of the electric light," Administrative Science Quarterly, vol. 46, no. 3, pp. 476-501, 2001.

doi: $10.2307 / 3094872$

[3] "Why HP is pruning the printers," Business Week, 2005.

Available:

http://www.businessweek.com/stories/2005-04-2 7/why-hp-is-pruning-the-printers

[4] T. Modis, "Genetic re-engineering of corporations," Technological Forecasting and Social Change, vol. 56, no. 2, pp. 107-118, 1997. doi: 10.1016/S0040-1625(97)00076-0

[5] R. Leoncini, "Segmentation and increasing returns in the evolutionary dynamics of competing techniques," Metroeconomica, vol. 52, no. 2, pp. 217-237, 2001.

doi: 10.1111/1467-999X.00115

[6] Z. Zhang, "Mutualism or cooperation among competitors promotes coexistence and competitive ability," Ecological Modelling, vol. 164, no. 2-3, pp. 271-282, 2003.

doi: 10.1016/S0304-3800(03)00069-3

[7] M. E. Ferguson and L. B. Toktay, "The effect of competition on recovery strategies," Production and Operations Management, vol. 15, no. 3, pp. 351-368, 2006

doi: 10.1111/i.1937-5956.2006.tb00250.x

[8] P. Majumder and H. Groenevelt, "Competition in remanufacturing," Production and Operations Management, vol. 10, no. 2, pp. 125-141, 2001. doi: 10.1111/j.1937-5956.2001.tb00074.x

[9] Y. J. Chen and J.B. Sheu, "Pursuing Extended Producer Responsibility in the Context of EIPs by a Hotelling Model," Journal of Cleaner Production, vol. 15, pp. 52-157, 2013. doi: 10.1016/i.jclepro.2013.05.025

[10] V. Daniel R. Guide, R. H. Teunter, and L. N. Van Wassenhove, "Matching demand and supply to maximize profits from remanufacturing," Manufacturing \&amp; Service Operations Management, vol. 5, no. 4, pp. 303-316, 2003. doi: $10.1287 / \mathrm{msom} \cdot 5.4 .303 .24883$

[11] G. Ferrer and J. M. Swaminathan, "Managing new and remanufactured products," Management Science, vol. 52, no. 1, pp. 15-26, 2006. doi: $10.1287 / \mathrm{mnsc} .1050 .0465$

[12] Y. J. Chen, J.B. Sheu, and T.C. Lirn, "Fault Tolerance Modeling for an E-waste Recycling Supply Chain," Transportation Research Part E: Logistics and Transportation Review, vol. 48, no. 5, pp. 897-906, 2012.

doi: 10.1016/i.tre.2012.02.005 
[13] M. L. Katz and C. Shapiro, "Systems competition and network effects," Journal of Economic Perspectives, vol. 8, no. 2, pp. 93-115, 1994. doi: $10.1257 /$ jep.8.2.93

[14] M. L. Katz and C. Shapiro, "Network externalities, competition, and compatibility," The American Economic Review, vol. 75, no. 3, pp. 424-440, 1985. doi: $10.2307 / 1814809$

[15] N. Economides, "The economics of networks," International Journal of Industrial Organization, vol. 14, no. 6, pp. 673-699, 1996. doi: 10.1016/0167-7187(96)01015-6

[16] G. Saloner and A. Shepard, "Adoption of technologies with network effects: An empirical examination of the adoption of automated teller machines," The RAND Journal of Economics, vol. 26, no. 3, pp. 479-501, 1995. doi: $10.2307 / 2555999$

[17] J. Farrell and G. Saloner, "Installed base and compatibility: Innovation, product preannouncements, and predation," The American Economic Review, vol. 76, no. 5, pp. 940-955, 1986. doi: $10.2307 / 1816461$

[18] M. T. Clements and H. Ohashi, "Indirect network effects and the product cycle: Video games in the U.S., 1994-2002," Journal of Industrial Economics, vol. 53, no. 4, pp. 515-542, 2005. doi: $\underline{10.2139 / \text { ssrn. } 500922}$
[19] N. Gandal, M. Kende, and R. Rob, "The dynamics of technological adoption in hardware/software systems: The case of compact disc players," The RAND Journal of Economics, vol. 31, no. 1, pp. 43-61, 2000.

doi: $10.2307 / 2601028$

[20] H. Nair, P. Chintagunta, and J.-P.Dubé, "Empirical analysis of indirect network effects in the market for personal digital assistants," Quantitative Marketing and Economics, vol. 2, no. 1, pp. 23-58, 2004.

doi: 10.1023/B:QMEC.0000017034.98302.44

[21] E. J. Dockner, S. Jørgensen, N. Van Long, and G. Sorger, Differential games in economics and management science. Cambridge, UK; New York: Cambridge University Press, 2000.

[22] X. He, A. Prasad, and S. P. Sethi, "Cooperative advertising and pricing in a dynamic stochastic supply chain: Feedback Stackelberg strategies," Production and Operations Management, vol. 18, no. 1, pp. 78-94, 2009. doi: 10.1111/j.1937-5956.2009.01006.x

[23] L. Cabral, "Dynamic price competition with network effects," The Review of Economic Studies, vol. 78, no. 1, pp. 83-111, 2011. doi: $10.1093 / \mathrm{restud} / \mathrm{rdq} 007$ 